\title{
Multi-target tracking with PHD filter using Doppler-only measurements
}

Mehmet B. Guldogan, David Lindgren, Fredrik Gustafsson, Hans Habberstad and Umut Orguner

Linköping University Post Print

\section{Tweet}

N.B.: When citing this work, cite the original article.

Original Publication:

Mehmet B. Guldogan, David Lindgren, Fredrik Gustafsson, Hans Habberstad and Umut Orguner, Multi-target tracking with PHD filter using Doppler-only measurements, 2014, Digital signal processing (Print), (27), , 1-11.

http://dx.doi.org/10.1016/j.dsp.2014.01.009

Copyright: Elsevier http://www.elsevier.com/

Postprint available at: Linköping University Electronic Press http://urn.kb.se/resolve?urn=urn:nbn:se:liu:diva-106861 


\title{
Multi-Target Tracking with PHD Filter using Doppler-Only Measurements
}

\author{
Mehmet B. Guldogan ${ }^{a, *}$, David Lindgren ${ }^{b}$, Fredrik Gustafsson ${ }^{c}$, Hans Habberstad ${ }^{b}$, Umut Orguner $^{d}$ \\ ${ }^{a}$ Department of Electrical and Electronics Engineering, Turgut Ozal University, Ankara, Turkey \\ ${ }^{b}$ Swedish Defence Research Agency (FOI), Linköping, 58183 Sweden \\ ${ }^{c}$ Department of Electrical Engineering, Linköping University, Linköping, 58183 Sweden \\ ${ }^{d}$ Department of Electrical and Electronics Engineering, Middle East Technical University, Ankara, Turkey
}

\begin{abstract}
In this paper, we address the problem of multi-target detection and tracking over a network of separately located Doppler-shift measuring sensors. For this challenging problem, we propose to use the probability hypothesis density (PHD) filter and present two implementations of the PHD filter, namely the sequential Monte Carlo PHD (SMC-PHD) and the Gaussian mixture PHD (GM-PHD) filters. Performances of both filters are carefully studied and compared for the considered challenging tracking problem. Simulation results show that both PHD filter implementations, successfully tracks multiple targets using only Doppler shift measurements. Moreover, as a proof-of-concept, an experimental setup consisting of a network of microphones and a loudspeaker was prepared. Experimental study results reveal that it is possible to track multiple ground targets using acoustic Doppler shift measurements in a passive multi-static scenario. We observed that the GM-PHD is more effective, efficient and easy to implement than the SMC-PHD filter.
\end{abstract}

Keywords: Random sets, Multi-target tracking, Probability hypothesis density filter, Doppler measurements, Gaussian mixture, Sequential Monte Carlo.

\section{Introduction}

Multi-static radar/sonar systems making use of cooperative/noncooperative transmitters have attracted interest of researchers working in different fields [1]. Modern multi-static systems consist of multiple transmitter and receiver sites each collecting several independent target measurements, such as the time-of-arrival, direction-of-arrival and Doppler shift of the reflected signals. At the fusion center, these measurements are then combined to estimate the target state. For target surveillance, multi-static passive radar systems exploit illuminators of opportunity like FM radio transmitters, digital audio/video broadcasters, WiMAX systems and global system for mobilecommunication (GSM) base stations [2], [3], [4], [5]. Passive radar systems provide crucial advantages over active systems: no frequency allocation problem, receivers are hidden for a possible jamming, energy saving and much lower costs. Especially, GSM-based passive radar systems have attracted tremendous research interest and they are considered to be used practically for surveillance [5], [6], [7]. These systems have several distinct advantages. Firstly, GSM base stations provide global coverage. Secondly, multiple base stations can be utilized in a multi-static passive radar network to improve the overall performance and robustness. Although the GSM waveform has poor range resolution, it can achieve good Doppler resolution,

\footnotetext{
${ }^{*}$ Corresponding author.

${ }^{a, d}$ This work was started at Linköping University.

Email address: bguldogan@turgutozal.edu.tr (Mehmet B. Guldogan ${ }^{a,}$ )
}

which makes the GSM-based passive radar suitable for Doppler detection and tracking.

Localization and tracking of a moving target using only Doppler shift measurements is actually an old problem studied in different contexts [8], [9], [10]. However, analysis of multi-static passive systems that use Doppler only measurement has not been fully investigated yet. Some of the studies in the literature mainly concentrate on the static estimation solutions, observability analysis of the target using Doppler-only measurements and optimal positioning of the passive system [11], [12], [13], [14], [15]. Moreover, recently, tracking of moving targets using a Doppler-shift measuring sensor network has gained interest from researchers and mostly considered in multi-static passive radar framework [16], [17], [18], [19]. Two main reasons behind this interest is; firstly passive radar systems provide crucial advantages over active systems, secondly Doppler measuring sensors are inexpensive and no hardware array is required unlike the direction-of-arrival measuring arrays. However, tracking using Doppler-only measurements is not an easy problem due to several reasons: 1-)since Doppleronly measurements are uninformative, target state remains unobservable before collecting at least three Doppler measurements from sensors with different locations, 2-) since Doppler measurements are typically accurate and initially target state is unobservable, initial measurement updates are weighted significantly and, thus, low-complexity nonlinear filters diverge and also special care should be given to avoid sample impoverishment when using particle implementations, 3-)mentioned two problems get worse if the prior distribution covers the whole 
surveillance volume (i.e. too big covariance values for location and velocity), which is the case in most of the practical applications, in the state space [20], [21], [22].

In this work, we propose to use the probability hypothesis density (PHD) filter, which is based on the random finite sets (RFS) framework [23], [24]. The PHD filter, propagates the first-order statistical moment of the RFS of states in time and avoids the combinatorial data association problem. There are two implementations of the PHD filter; one is using sequential Monte Carlo (SMC) method other one is using Gaussian mixtures (GM). Each implementation method has its own pros and cons [23]. GM implementation is very popular because it provides a closed form analytic solution to PHD recursions under linear Gaussian target dynamics and measurement models. Moreover, contrary to SMC implementation, GM implementation provides reliable state estimates extracted from the posterior intensity in an easier and efficient way [25]. Alternatively, SMC implementation imposes no such restrictions and has the ability of handling nonlinear target dynamics and measurement models. It can be said that SMC implementation is a more general framework for PHD recursions. On the other hand, its performance is affected by different kind of problems in reality [26], [27], [28]. Therefore, in general, GM based approach is easier, effective and more intuitive.

The novelty of this work is twofold; firstly we present the performances of both the SMC-PHD and GM-PHD filters in tracking multiple non-cooperative targets using a passive Doppler-shift measuring sensor network. Clutter, missed detections and multi-static Doppler variances are incorporated into a realistic multi-target scenario. Secondly, additional to the simulation analysis, we provide a proof-of-concept study to show the feasibility of tracking multiple targets using a passive acoustic microphone network which provides Doppler shift measurements. For this purpose, an experimental setup consisting of three microphones and a loudspeaker (LS) was configured. Non-cooperative transmissions from the LS (i.e. illuminator of opportunity) are exploited by non-directional separately located microphones (i.e. Doppler measuring sensors). The LS is directed towards the road and continuously transmits pure sinusoids at a known certain frequency. Reflections from moving vehicles on the road are received by each microphone and microphone outputs are connected to a main storage unit through cables to be processed. Doppler shift measurements from each microphone are fed to the tracker.

The organization of the paper is as follows. Mathematical formulation of the problem and the measurement model are presented in Section 2. Section 3 presents RFS formulation of multi-target tracking and the PHD filter. Section 4 and 5 provide SMC-PHD and GM-PHD formulations, respectively. Simulation results are presented in Section 6. Details of the acoustic field trials and results are given in Section 7. Lastly, conclusion is given in Section 8.

\section{Sensor and Target Model}

The scenario in this work is as follows: An illuminator of opportunity (TX), constantly transmits signal with a known carrier

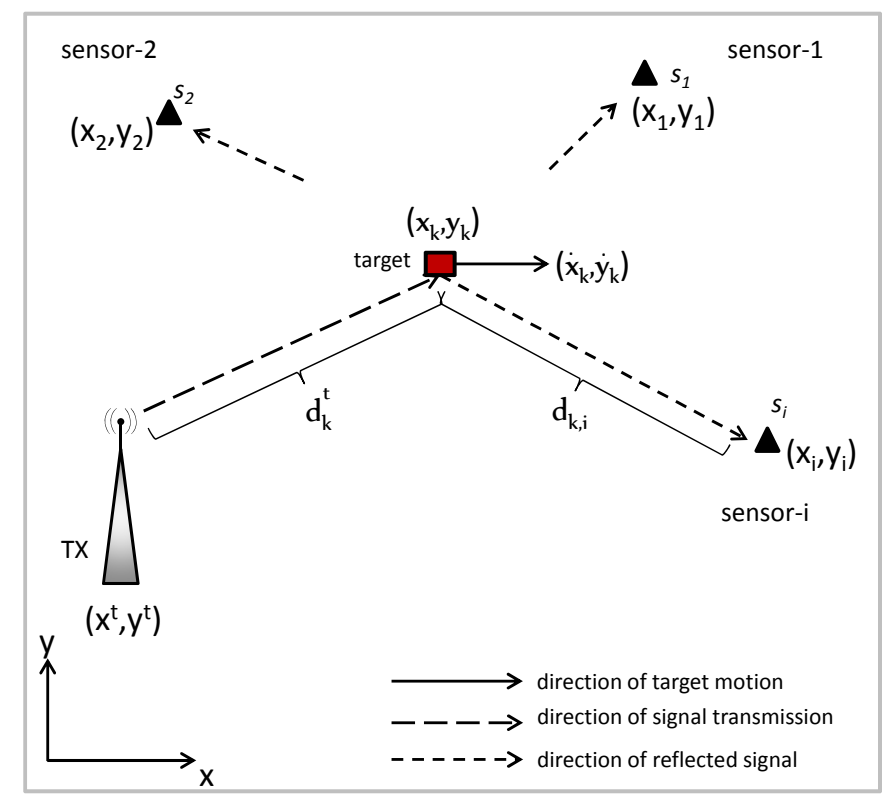

Figure 1: Multi-static geometry. Transmitted signal from an illuminator of opportunity TX is reflected from a detected moving target and received by the each Doppler shift measuring sensors (black triangles). $s_{i}: i=1, \ldots, N_{s}$.

frequency, $f_{c}$, as in Fig 1. The transmitted signal is reflected from moving targets in the analyzed area. These reflections are received by each Doppler shift measuring sensors. It is assumed that location of the transmitter and the sensors are known to the fusion center and each sensor sends its measurement to the fusion center. The state vector of a target, takes a value in the state space $\mathcal{X} \subseteq \mathbb{R}^{n_{\mathbf{x}}}$, at time $k$ is

$$
\mathbf{x}_{k}=\left[x_{k}, y_{k}, \dot{x}_{k}, \dot{y}_{k}\right]^{T},
$$

where $\left[x_{k}, y_{k}\right]$ is the position, $\left[\dot{x}_{k}, \dot{y}_{k}\right]$ is the velocity of the target and $T$ denotes transpose operation. The target dynamic is modeled by linear Gaussian constant velocity model [29]:

$$
\mathbf{x}_{k}=\mathbf{F} \mathbf{x}_{k-1}+\mathbf{v}_{k}
$$

where $\mathbf{F}$ is the state transition matrix given as,

$$
\mathbf{F}=\left[\begin{array}{cc}
\mathbf{I}_{2} & \Delta \mathbf{I}_{2} \\
\mathbf{0}_{2} & \mathbf{I}_{2}
\end{array}\right],
$$

$\mathbf{v}_{k} \sim \mathcal{N}(\mathbf{v} ; \mathbf{0}, \mathbf{Q})$ is the white Gaussian process noise, $\mathbf{Q}$ is the process noise covariance given as,

$$
\mathbf{Q}=\sigma_{\mathbf{v}}^{2}\left[\begin{array}{cc}
\frac{\Delta^{3}}{3} \mathbf{I}_{2} & \frac{\Delta^{2}}{2} \mathbf{I}_{2} \\
\frac{\Delta^{2}}{2} \mathbf{I}_{2} & \Delta \mathbf{I}_{2}
\end{array}\right],
$$

$\Delta$ is the sampling interval, $k$ is the discrete time index, $\sigma_{\mathbf{v}}$ is the standard deviation of the process noise, $\mathbf{I}_{n}$ and $\mathbf{0}_{n}$ denote $n \times n$ identity and zero matrices, respectively.

Bi-static Doppler shift measurements are collected by each sensor, $i=1, \ldots, N_{s}$, in the area. Motion components of the target in the directions of the transmitter and the receiver together cause Doppler shift. The measured bi-static Doppler shift, takes 
a value in the measurement space $\mathcal{Z} \subseteq \mathbb{R}^{n_{z}}$, by the $i$-th sensor located at $\left[\mathrm{x}_{i}, \mathrm{y}_{i}\right]$ is given by;

$$
z_{k, i}=h_{i}\left(\mathbf{x}_{k}\right)+\varepsilon_{k, i}
$$

where

$$
h_{i}\left(\mathbf{x}_{k}\right)=-\left[\frac{\dot{d}_{k}^{t}}{\lambda}+\frac{\dot{d}_{k, i}}{\lambda}\right],
$$

is the Doppler shift, $\lambda$ is the wavelength of the transmitted signal and $\varepsilon_{k, i}$ is measurement noise in sensor $i, \varepsilon_{k, i} \sim \mathcal{N}\left(\varepsilon ; 0, \sigma_{\varepsilon}^{2}\right)$. Distance between the transmitter and the target, $d_{k}^{t}$, at time $k$ is defined as

$$
d_{k}^{t}=\sqrt{\left(x_{k}-\mathrm{x}^{t}\right)^{2}+\left(y_{k}-\mathrm{y}^{t}\right)^{2}},
$$

and the distance between the target and the $i$-th sensor, $d_{k, i}$, at time $k$ is

$$
d_{k, i}=\sqrt{\left(x_{k}-\mathrm{x}_{i}\right)^{2}+\left(y_{k}-\mathrm{y}_{i}\right)^{2}} .
$$

Total derivatives of $d_{k}^{t}$ and $d_{k, i}$ can be respectively written as

$$
\begin{gathered}
\dot{d}_{k}^{t}=\frac{\dot{x}_{k}\left(x_{k}-\mathrm{x}^{t}\right)+\dot{y}_{k}\left(y_{k}-\mathrm{y}^{t}\right)}{d_{k}^{t}}, \\
\dot{d}_{k, i}=\frac{\dot{x}_{k}\left(x_{k}-\mathrm{x}_{i}\right)+\dot{y}_{k}\left(y_{k}-\mathrm{y}_{i}\right)}{d_{k, i}},
\end{gathered}
$$

We also here provide Jacobian of $h_{i}\left(\mathbf{x}_{k}\right), \mathbf{H}_{k, i}$, to be used in the filtering as;

$$
\mathbf{H}_{k, i}=\left[\begin{array}{llll}
\frac{\partial h_{i}\left(\mathbf{x}_{k}\right)}{\partial x_{k}} & \frac{\partial h_{i}\left(\mathbf{x}_{k}\right)}{\partial y_{k}} & \frac{\partial h_{i}\left(\mathbf{x}_{k}\right)}{\partial \dot{x}_{k}} & \frac{\partial h_{i}\left(\mathbf{x}_{k}\right)}{\partial \dot{y}_{k}}
\end{array}\right]
$$

and each of its elements are

$$
\begin{aligned}
& \frac{\partial h_{i}\left(\mathbf{x}_{k}\right)}{\partial x_{k}}=-\left\{\frac{\dot{x}_{k} d_{k}^{t}-\left(x_{k}-\mathrm{x}^{t}\right) \dot{d}_{k}^{t}}{\lambda d_{k}^{2}}\right\}-\left\{\frac{\dot{x}_{k} d_{k, i}-\left(x_{k}-\mathrm{x}_{i}\right) \dot{d}_{k, i}}{\lambda d_{k, i}^{2}}\right\} \\
& \frac{\partial h_{i}\left(\mathbf{x}_{k}\right)}{\partial y_{k}}=-\left\{\frac{\dot{y}_{k} d_{k}^{t}-\left(y_{k}-\mathrm{y}^{t}\right) \dot{d}_{k}^{t}}{\lambda d_{k}^{t}}\right\}-\left\{\frac{\dot{y}_{k} d_{k, i}-\left(y_{k}-\mathrm{y}_{i}\right) \dot{d}_{k, i}}{\lambda d_{k, i}^{2}}\right\} \\
& \frac{\partial h_{i}\left(\mathbf{x}_{k}\right)}{\partial \dot{x}_{k}}=-\left\{\frac{\left(x_{k}-\mathrm{x}^{t}\right)}{\lambda d_{k}^{t}}+\frac{\left(x_{k}-\mathrm{x}_{i}\right)}{\lambda d_{k, i}}\right\} \\
& \frac{\partial h_{i}\left(\mathbf{x}_{k}\right)}{\partial \dot{y}_{k}}=-\left\{\frac{\left(y_{k}-\mathrm{y}^{t}\right)}{\lambda d_{k}^{t}}+\frac{\left(y_{k}-\mathrm{y}_{i}\right)}{\lambda d_{k, i}}\right\}
\end{aligned}
$$

In the next section, we provide important points of RFS based multi-target filtering.

\section{Random Finite Sets (RFS) Based Filtering}

The RFS framework for multiple target tracking proposed by Mahler combines the problems of combinatorial data association, detection, classification and target tracking within a unified compact Bayesian paradigm [23], [24]. In the following subsections, basic RFS notation, multiple target generalization of the Bayes filter and its first order approximation PHD filter are described.

\subsection{RFS Formulation}

The RFS approach treats the collection of the individual targets and individual measurements as a set-valued state and setvalued measurement, respectively, as

$$
\begin{aligned}
& X_{k}=\left\{\mathbf{x}_{k, 1}, \ldots, \mathbf{x}_{k, M(k)}\right\} \in \mathcal{F}(\mathcal{X}) \\
& Z_{k}=\left\{z_{k, 1}, \ldots, z_{k, N(k)}\right\} \in \mathcal{F}(\mathcal{Z})
\end{aligned}
$$

where $M(k)$ is the number of targets at time $k, N(k)$ is the number of measurements at time $k, \mathcal{F}(\mathcal{X})$ and $\mathcal{F}(\mathcal{Z})$ are the set of all possible finite subsets of state space $\mathcal{X}$ and measurement space $\mathcal{Z}$, respectively. An RFS model for the time evolution of a multi-target state $X_{k-1}$ at time $k-1$ to the multi-target state $X_{k}$ at time $k$ is defined as

$$
X_{k}=\left[\bigcup_{\zeta \in X_{k-1}} S_{k \mid k-1}(\zeta)\right] \cup \Gamma_{k},
$$

where $S_{k \mid k-1}(\zeta)$ is the RFS of surviving targets from previous state $\zeta$ at time $k$ and $\Gamma_{k}$ is the RFS of spontaneous target births at time $k$. The RFS measurement model for a multi-target state $X_{k}$ at time $k$ can be written as

$$
Z_{k}=K_{k} \cup\left[\bigcup_{\mathbf{x} \in X_{k}} \Theta_{k}(\mathbf{x})\right]
$$

where $K_{k}$ is the RFS of clutter or false measurements, $\Theta_{k}(\mathbf{x})$ is the RFS of multi-target state originated measurements, which can take values either $z_{k}$ if target is detected, or $\emptyset$ if target is not detected.

\subsection{Multi-target Filtering}

Having very briefly summarized some key points of the RFS framework, we can define the RFS based multi-target Bayes filter. The optimal multi-target Bayes filter propagates the multitarget posterior density $p_{k}\left(\cdot \mid Z_{1: k}\right)$ conditioned on the sets of measurements up to time $k, Z_{1: k}$, in time with the following recursion

$$
\begin{gathered}
p_{k \mid k-1}\left(X_{k} \mid Z_{1: k-1}\right)=\int f_{k \mid k-1}\left(X_{k} \mid X\right) p_{k-1}\left(X \mid Z_{1: k-1}\right) \delta X, \\
p_{k}\left(X_{k} \mid Z_{1: k}\right)=\frac{g_{k}\left(Z_{k} \mid X_{k}\right) p_{k \mid k-1}\left(X_{k} \mid Z_{1: k-1}\right)}{\int g_{k}\left(Z_{k} \mid X\right) p_{k \mid k-1}\left(X \mid Z_{1: k-1}\right) \delta X},
\end{gathered}
$$

where $f_{k \mid k-1}$ is the multi-target transition density, $g_{k}\left(Z_{k} \mid X_{k}\right)$ is the multi-target likelihood and integrals are set integrals defined in [24], [23]. The multi-target Bayes recursion involves multiple integrals and the complexity of computing it grows exponentially with the number of targets. Therefore, it is not practical for scenarios where there exist more than a few targets.

\subsection{The Probability Hypothesis Density (PHD) Filter}

To alleviate the computational burden in calculating the optimal filter given above, the PHD filter was proposed as a practical suboptimal alternative [24]. The PHD filter propagates the first-order statistical moment of the posterior multi-target state, 
instead of propagating the multi-target posterior density. Consider that, intensities associated with the multi-target posterior density $p_{k}$ and the multitarget predicted density $p_{k \mid k-1}$ in the optimal multi-target Bayes recursion are represented with $v_{k}$ and $v_{k \mid k-1}$ respectively. The PHD recursion is defined as

$$
v_{k \mid k-1}(\mathbf{x})=\int p_{s} f_{k \mid k-1}(\mathbf{x} \mid \zeta) v_{k-1}(\boldsymbol{\zeta}) d \zeta+\gamma_{k}(\mathbf{x}),
$$

$v_{k}(\mathbf{x})=\left(1-p_{D}\right) v_{k \mid k-1}(\mathbf{x})+\sum_{z \in Z_{k}} \frac{p_{D} g_{k}(z \mid \mathbf{x}) v_{k \mid k-1}(\mathbf{x})}{\kappa_{k}(z)+\int p_{D} g_{k}(z \mid \boldsymbol{\xi}) v_{k \mid k-1}(\boldsymbol{\xi}) d \boldsymbol{\xi}}$,

where $p_{s}$ is the probability of target survival, $\gamma_{k}(\mathbf{x})$ is the intensity of spontaneous birth RFS at time $k, p_{D}$ is the probability of target detection and $\kappa_{k}(z)$ is the intensity of clutter RFS at time $k$.

PHD filters can be implemented either by using GM [25] or SMC [30] based methods. In the next two sections, we describe main steps of these two approaches.

\section{The Sequential Monte Carlo PHD (SMC-PHD) Filter}

In this section, we describe key steps of the SMC implementation of the PHD filter. Details and algorithmic flow of the SMC implementation can be found in [31], [32]. Basically, random distributed particles are used to approximate the intensity function. Let at time $k-1$, we have

$$
v_{k-1}(\mathbf{x})=\sum_{i=1}^{J_{k-1}^{p}} w_{k-1, p}^{(i)} \delta_{\mathbf{x}_{k-1, p}^{(i)}}(\mathbf{x})+\sum_{i=1}^{J_{k-1}^{b}} w_{k-1, b}^{(i)} \delta_{\mathbf{x}_{k-1, b}^{(i)}}(\mathbf{x})
$$

where $\delta_{\mathbf{x}}$ is the Dirac delta function, $J_{k-1}^{p}$ and $J_{k-1}^{b}$ are the number of persistent target and newborn target particles, respectively, and $\left\{\left(w_{k-1, p}^{(i)}, \mathbf{x}_{k-1, p}^{(i)}\right)\right\}_{i=1}^{J_{k-1}^{p}}$ and $\left\{\left(w_{k-1, b}^{(i)}, \mathbf{x}_{k-1, b}^{(i)}\right)\right\}_{i=1}^{J_{k-1}^{b}}$ are the persistent and newborn weighted particle sets, respectively. In a more compact form, intensity function at time $k-1$ can be written as

$$
v_{k-1}(\mathbf{x})=\sum_{i=1}^{J_{k-1}} w_{k-1}^{(i)} \delta_{\mathbf{x}_{k-1}^{(i)}}(\mathbf{x})
$$

where $\left\{\left(w_{k-1}^{(i)}, \mathbf{x}_{k-1}^{(i)}\right)\right\}_{i=1}^{J_{k-1}}$ is the union of the two particle sets;

$$
\left\{\left(w_{k-1}^{(i)}, \mathbf{x}_{k-1}^{(i)}\right)\right\}_{i=1}^{J_{k-1}}=\left\{\left(w_{k-1, p}^{(i)}, \mathbf{x}_{k-1, p}^{(i)}\right)\right\}_{i=1}^{J_{k-1}^{p}} \cup\left\{\left(w_{k-1, b}^{(i)}, \mathbf{x}_{k-1, b}^{(i)}\right)\right\}_{i=1}^{J_{k-1}^{b}} .
$$

The predicted intensity function can be approximated by the particle set;

$$
v_{k \mid k-1, p}(\mathbf{x})=\sum_{i=1}^{J_{k-1}} w_{k \mid k-1, p}^{(i)} \delta_{\mathbf{x}_{k \mid k-1, p}^{(i)}}(\mathbf{x})
$$

where

$$
\begin{aligned}
\mathbf{x}_{k \mid k-1, p}^{(i)} & =\mathbf{F} \mathbf{x}_{k-1}^{(i)}, \\
w_{k \mid k-1, p}^{(i)} & =p_{s} w_{k-1}^{(i)} .
\end{aligned}
$$

In order to approximate $\gamma_{k}(\mathbf{x})$, which should cover all the surveillance area unless a prior knowledge exists, a massive number of particles is required. Moreover majority of these particles will be thrown in the resampling step. To handle this problem and demonstrate a more realistic scenario, we prefer to generate birth particles adaptively, details of which are given in [32]. In this scheme, for each $z \in Z_{k}$, a set of $M_{b}$ particles are generated in the region of the state space where the inner product $\int g_{k}(z, \mathbf{x}) \gamma_{k}(\mathbf{x}) d \mathbf{x}$ will have high values. Therefore, $J_{k}^{b}=M_{b} \cdot\left|Z_{k}\right|$ number of newborn particles are generated with uniform weights, i.e.,

$$
w_{k \mid k-1, b}^{(i)}=\frac{v_{k \mid k-1}^{b}}{J_{k}^{b}},
$$

where $v_{k \mid k-1}^{b}$ represents the prior expected number of target births. In short, target birth intensity is formed by an equally weighted mixture of birth densities $b(\mathbf{x} \mid z)$, for $z \in Z_{k}$. In the update step of the SMC-PHD filter, weights of persistent target particles are updated as;

$$
w_{k \mid k, p}^{(i)}=\left(1-p_{D}\right) w_{k \mid k-1, p}^{(i)}+\sum_{z \in Z_{k}} \frac{p_{D} g_{k}\left(z \mid \mathbf{x}_{k \mid k-1, p}^{(i)}\right) w_{k \mid k-1, p}^{(i)}}{\mathcal{L}(z)},
$$

where

$$
\mathcal{L}(z)=\kappa_{k}(z)+\sum_{i=1}^{J_{k}^{b}} w_{k \mid k-1, b}^{(i)}+\sum_{i=1}^{J_{k-1}} p_{D} g_{k}\left(z \mid \mathbf{x}_{k \mid k-1, p}^{(i)}\right) w_{k \mid k-1, p}^{(i)} .
$$

In a similar way, weights of the newborn target particles are updated by

$$
w_{k \mid k, b}^{(i)}=\sum_{z \in Z_{k}} \frac{w_{k \mid k-1, b}^{(i)}}{\mathcal{L}(z)} .
$$

Following the update step, resampling step takes place. Intensity function of persistent and birth target intensities are resampled $J_{k}^{p}$ and $J_{k}^{b}$ times, respectively [32], [27]. Number of persistent target particles, $J_{k}^{p}$, is determined by

$$
J_{k}^{p}=\left\lfloor M_{p} \sum_{i=1}^{J_{k-1}} w_{k \mid k, p}^{(i)}\right\rceil=\left\lfloor M_{p} \hat{v}_{k}^{p}\right\rceil,
$$

where $M_{p}$ is the number of particles per persistent target, $L \cdot 7$ denotes the nearest integer and $\hat{v}_{k}^{p}$ is the estimate of the number of persistent targets. Weights of resampled particles are set to $w_{k, p}^{(i)}=\hat{v}_{k}^{p} / J_{k}^{p}$. Similarly, expected number of newborn targets at time $k$ is $\hat{v}_{k}^{b}=\sum_{i=1}^{J_{k}^{b}} w_{k \mid k, b}^{(i)}$ and weights of resampled particles are set to $w_{k, b}^{(i)}=\hat{v}_{k}^{b} / J_{k}^{b}$. Finally, particle set $\left\{\left(w_{k, p}^{(i)}, \mathbf{x}_{k, p}^{(i)}\right)\right\}_{i=1}^{J_{k}^{p}}$ and cardinality estimate $\hat{v}_{k}^{p}$ are reported as filter outputs. Particle grouping and state estimation are performed efficiently and accurately using the procedure detailed in [30], [32].

\section{The Gaussian Mixture PHD (GM-PHD) Filter}

Vo et al. derived a closed-form solution to the PHD filter, called as the GM-PHD under linear Gaussian multi-target models in [25]. The GM-PHD filter has been successfully used in 
many different applications [16], [33], [34], [35], [36], [37]. Here, it is important to note that, in these applications target models are nonlinear. In order to accommodate nonlinear Gaussian models, an adaptation of the GM-PHD filter (called as EKPHD) is provided based on the idea of extended Kalman (EKF) filter, where local linearizations of the nonlinear measurement function $h(\mathbf{x})$ (i.e. $\mathbf{H}_{k}$ defined in (11)) is used [25]. In this work, we used the mentioned adaptation to handle nonlinearities in measurement model in (6).

Assume that the posterior intensity at time $k-1$ can be written as a sum of Gaussian components with different weights, means and covariances as

$$
\begin{aligned}
v_{k-1}(\mathbf{x}) & =\sum_{i=1}^{J_{k-1}^{p}} w_{k-1, p}^{(i)} \mathcal{N}\left(\mathbf{x} ; \mathbf{m}_{k-1, p}^{(i)}, \mathbf{P}_{k-1, p}^{(i)}\right) \\
& +\sum_{i=1}^{J_{k-1}^{b}} w_{k-1, b}^{(i)} \mathcal{N}\left(\mathbf{x} ; \mathbf{m}_{k-1, b}^{(i)}, \mathbf{P}_{k-1, b}^{(i)}\right)
\end{aligned}
$$

where $J_{k-1}^{p}$ and $J_{k-1}^{b}$ are the number of persistent target and newborn target particles, respectively, and $\left\{\left(w_{k-1, p}^{(i)}, \mathbf{m}_{k-1, p}^{(i)}, \mathbf{P}_{k-1, p}^{(i)}\right)\right\}_{i=1}^{J_{k-1}^{p}}$ and $\left\{\left(w_{k-1, b}^{(i)}, \mathbf{m}_{k-1, b}^{(i)}, \mathbf{P}_{k-1, b}^{(i)}\right)\right\}_{i=1}^{J_{k-1}^{b}}$ are the persistent and newborn weighted GMs, respectively. In a more compact form, intensity function at time $k-1$ can be written as

$$
v_{k-1}(\mathbf{x})=\sum_{i=1}^{J_{k-1}} w_{k-1}^{(i)} \mathcal{N}\left(\mathbf{x} ; \mathbf{m}_{k-1}^{(i)}, \mathbf{P}_{k-1}^{(i)}\right),
$$

where $\left\{\left(w_{k-1}^{(i)}, \mathbf{m}_{k-1}^{(i)}, \mathbf{P}_{k-1}^{(i)}\right)\right\}_{i=1}^{J_{k-1}}$ is the union of the two GMs;

$$
\begin{aligned}
\left\{\left(w_{k-1}^{(i)}, \mathbf{m}_{k-1}^{(i)}, \mathbf{P}_{k-1}^{(i)}\right)\right\}_{i=1}^{J_{k-1}} & =\left\{\left(w_{k-1, p}^{(i)}, \mathbf{m}_{k-1, p}^{(i)}, \mathbf{P}_{k-1, p}^{(i)}\right)\right\}_{i=1}^{J_{k-1}^{p}} \\
& \cup\left\{\left(w_{k-1, b}^{(i)}, \mathbf{m}_{k-1, b}^{(i)}, \mathbf{P}_{k-1, b}^{(i)}\right)\right\}_{i=1}^{J_{k-1}^{b}}
\end{aligned}
$$

The predicted intensity function can be written in the form of a GM as;

$$
v_{k \mid k-1, p}(\mathbf{x})=\sum_{i=1}^{J_{k-1}} w_{k \mid k-1, p}^{(i)} \mathcal{N}\left(\mathbf{x} ; \mathbf{m}_{k \mid k-1, p}^{(i)}, \mathbf{P}_{k \mid k-1, p}^{(i)}\right),
$$

where

$$
\begin{aligned}
& \mathbf{m}_{k \mid k-1, p}^{(i)}=\mathbf{F} \mathbf{m}_{k-1}^{(i)} \\
& w_{k \mid k-1, p}^{(i)}=p_{s} w_{k-1}^{(i)} \\
\mathbf{P}_{k \mid k-1, p}^{(i)} & =\mathbf{Q}+\mathbf{F P}_{k-1}^{(i)} \mathbf{F}^{T} .
\end{aligned}
$$

Due to similar considerations in previous section, birth Gaussian components are generated adaptively. For each $z \in Z_{k}$, a GM is created with $M_{b}$ components. In total, $J_{k}^{b}=M_{b} \cdot\left|Z_{k}\right|$ number of Gaussian components are generated with uniform weights, i.e. $w_{k \mid k-1, b}^{(i)}=\frac{v_{k \mid k-1}^{b}}{J_{k}^{b}}$. The posterior intensity at time $k$ is also a GM and can be written as

$$
\begin{aligned}
v_{k \mid k, p}(\mathbf{x}) & =\left(1-p_{D}\right) v_{k \mid k-1, p}(\mathbf{x}) \\
& +\sum_{z \in Z_{k}} \sum_{i=1}^{J_{k-1}} w_{k \mid k, p}^{(i)}(z) \mathcal{N}\left(\mathbf{x} ; \mathbf{m}_{k \mid k, p}^{(i)}(z), \mathbf{P}_{k \mid k, p}^{(i)}\right),
\end{aligned}
$$

where

$$
\begin{array}{r}
w_{k \mid k, p}^{(i)}=\left(1-p_{D}\right) w_{k \mid k-1, p}^{(i)}+\sum_{z \in Z_{k}} \frac{p_{D} w_{k \mid k-1, p}^{(i)} q_{k}^{(i)}(z)}{\mathcal{L}(z)} \\
\mathcal{L}(z)=\kappa_{k}(z)+\sum_{i=1}^{J_{k}^{b}} w_{k \mid k-1, b}^{(i)}+\sum_{i=1}^{J_{k-1}} p_{D} w_{k \mid k-1, p}^{(i)} q_{k}^{(i)}(z) \\
q_{k}^{i}(z)=\mathcal{N}\left(z ; h\left(\mathbf{m}_{k \mid k-1, p}^{(i)}\right), \sigma_{\varepsilon}^{2}+\mathbf{H}_{k} \mathbf{P}_{k \mid k-1, p}^{(i)} \mathbf{H}_{k}^{T}\right) \\
\mathbf{m}_{k \mid k, p}^{(i)}(z)=\mathbf{m}_{k \mid k-1, p}^{(i)}+\mathbf{K}_{k}^{(i)}\left(z-h\left(\mathbf{m}_{k \mid k-1, p}^{(i)}\right)\right) \\
\mathbf{P}_{k \mid k, p}^{(i)}=\left[\mathbf{I}-\mathbf{K}_{k}^{(i)} \mathbf{H}_{k}\right] \mathbf{P}_{k \mid k-1, p}^{(i)}=\mathbf{P}_{k \mid k-1, p}^{(i)} \mathbf{H}_{k}^{T}\left(\mathbf{H}_{k} \mathbf{P}_{k \mid k-1, p}^{(i)} \mathbf{H}_{k}^{T}+\sigma_{\varepsilon}^{2}\right)^{-1} .
\end{array}
$$

In a similar way, weights of the newborn targets GM are given by

$$
w_{k \mid k, b}^{(i)}=\sum_{z \in Z_{k}} \frac{w_{k \mid k-1, b}^{(i)}}{\mathcal{L}(z)} .
$$

Accordingly, birth intensity is GM and can be written as

$$
v_{k \mid k, b}=\sum_{i=1}^{J_{k}^{b}} w_{k \mid k, b}^{(i)} \mathcal{N}\left(\mathbf{x} ; \mathbf{m}_{k \mid k, b}^{(i)}, \mathbf{P}_{k \mid k, b}^{(i)}\right)
$$

As time progresses, the number of Gaussian components increases and computational problems occur. To alleviate this problem, a simple pruning and merging can be used to decrease the number of Gaussian components propagated [25]. In order to extract multi-target states, means of the Gaussian components that have weights greater than some predefined threshold are selected and reported as filter output.

\section{Simulation Results}

In this section, we present simulation results of the SMCPHD [30], [32] and the GM-PHD [25] filters for tracking multiple targets using noisy multi-static Doppler shift measurements of separately distributed Doppler measuring sensors. The considered scenario contains two targets that are moving according to a model given in (2). True target trajectories denoted with black solid lines are seen in Fig. 2. Target-1 is born at discretetime $k=1$ and dies at discrete-time $k=65$ (i.e. $t=130$ ). Target-2 is born at discrete-time $k=15$ and dies at time $k=85$. Seven, $N_{s}=7$, Doppler-shift receiving sensors and a transmitter located as in Fig.2. Some parameters used in the simulation are: scan time or sampling interval $\Delta=2 \mathrm{~s}, \sigma_{\mathbf{v}}=0.2$, $p_{D}=0.96, p_{s}=0.99$. Total simulation time (i.e. duration of a single run of the scenario) is $T_{s i m}=170 \mathrm{~s}$. Carrier frequency is $f_{c}=950 \mathrm{MHz}$ and Doppler measurement standard deviation is chosen as $\sigma_{\varepsilon}=2 \mathrm{~Hz}$, which is a reasonable value used in real practical systems [38], [39]. At each scan, $\Delta$, a randomly chosen sensor reports its measurement set, $Z_{k}^{i}$. Namely, at a time only one sensor is active. This type of sensor measurement handling is practically more realistic compared to gathering all measurements from all sensors. At the same time, it makes the filtering problem more difficult. In other words, target state 


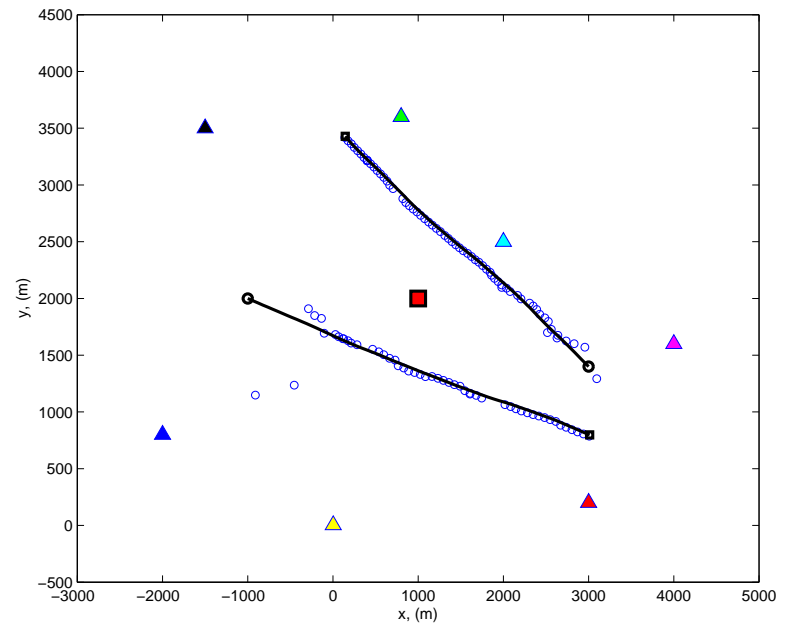

(a) Blue circles represent estimates of the GM-PHD filter for a single run.

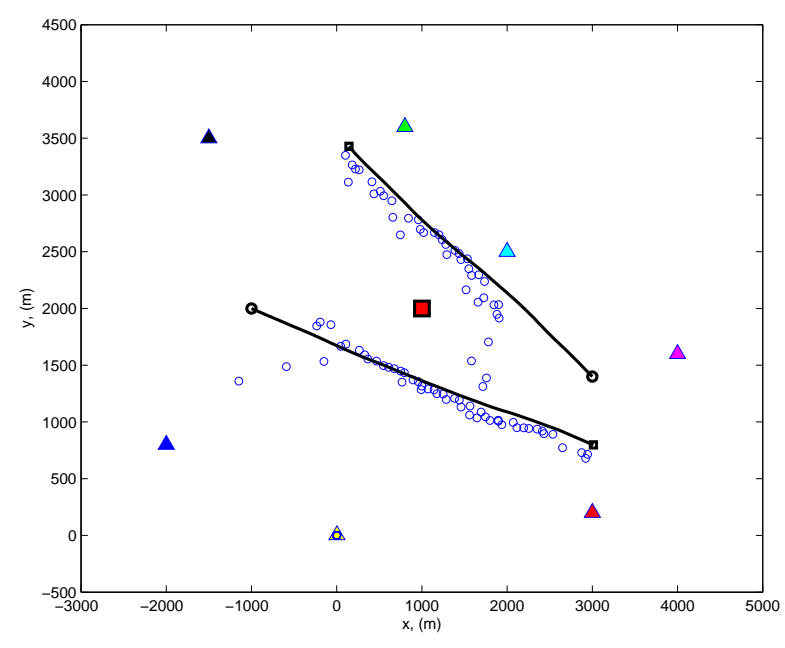

(b) Blue circles represent estimates of the SMC-PHD filter for a single run

Figure 2: Black solid lines denote true target trajectories. Transmitter is represented with a red shaded square. Sensors are color coded and represented with triangles. The black circles and squares mark the births and deaths of targets.

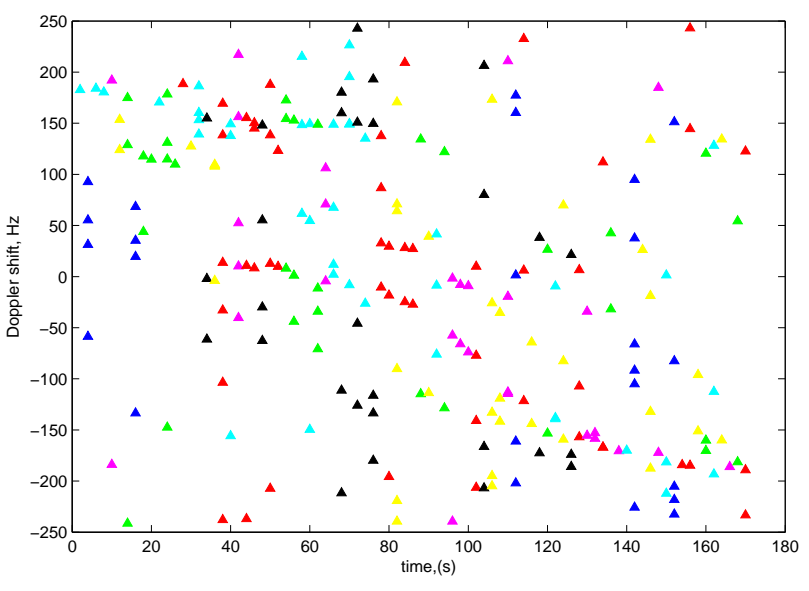

Figure 3: Doppler shift measurements obtained in a single run. Triangles are color coded compatible with the sensor colors in Fig. 2 .

remains unobservable before collecting at least three Doppler measurements from sensors with different locations [20], [19] [22]. The clutter is modeled as a Poisson RFS, $K_{k}$, having intensity

$$
\kappa_{k}(z)=\lambda_{c} V u(z),
$$

where $V=[-250,250] \mathrm{Hz}$ is the surveillance region, $\lambda_{c}=2 \times$ $10^{-3} \mathrm{~Hz}^{-1}$ is the average number of clutter returns and $u(z)$ is the uniform density over the surveillance region. In Fig. 3, Doppler-shift measurements obtained from randomly selected sensors are seen for a run of the scenario in Fig.2. Colors of measurements in Fig. 3 and sensor colors in Fig. 2 are matched (i.e. blue color coded sensor's Doppler-shift measurements are shown as blue triangles in Fig.3). As can be seen, there is no continuous Doppler pattern with same color.
GM-PHD filter parameters are chosen as follows. Maximum number of allowed Gaussian components is $J_{\max }=70$, truncation threshold is $T=10^{-5}$ and merging threshold is $U=4$. For each measurement $z \in Z_{k}, M_{b}=25$ birth Gaussian components are created and each of them are uniformly located on equidistance grid points covering whole surveillance area (e.g. in the considered scenario borders of the surveillance are is set as $6 \times 4 \mathrm{~km}^{2}$ ). Standard deviation of $x$ and $y$ parameters of each birth Gaussian component set to $500 \mathrm{~m}$. Velocity parameters, $\dot{x}$ and $\dot{y}$, of each birth Gaussian are selected to be compatible with Doppler-shift measurement $z$. The parameter $v_{k \mid k-1}^{b}=1 e-5$ is selected in order to have certain average number of newborn targets at each time.

Parameters used in SMC-PHD filtering are set as follows. Per persistent target $M_{p}=16000$ particles are generated. For each measurement $z \in Z_{k}, M_{b}=16000$ particles are generated by drawing samples from $\mathcal{N}(\mathbf{x} ; \mathbf{m}, \mathbf{P})$ and accepting the ones which has compatible velocity components with Doppler-shift measurement $z$. Parameters of the Gaussian distribution is selected to cover whole surveillance area; $\mathbf{m}=[1000 ; 2000 ; 0 ; 0]$, $\mathbf{P}=\operatorname{diag}\left[2500^{2} 2000^{2} 40^{2} 40^{2}\right]$. The prior expected number of target births is selected as $v_{k \mid k-1}^{b}=1 e-5$. As we mentioned before, since typically Doppler measurements are accurate, sample impoverishment problem occurs and particle diversity is lost in a short period of time. Therefore in order to increase particle diversity, after re-sampling step, Markov chain Monte Carlo (MCMC) move step is applied [26]. There exist also other approaches proposed in the literature to increase efficiency of particle implementations [27], [26], [28].

To evaluate filters' estimation error analysis, we use optimal subpattern assignment (OSPA) distance between the true multitarget and estimated state [40]. The OSPA distance between two finite sets, i.e., the state set $X=\left\{\mathbf{x}_{1}, \ldots \mathbf{x}_{n}\right\}$ and the ground 


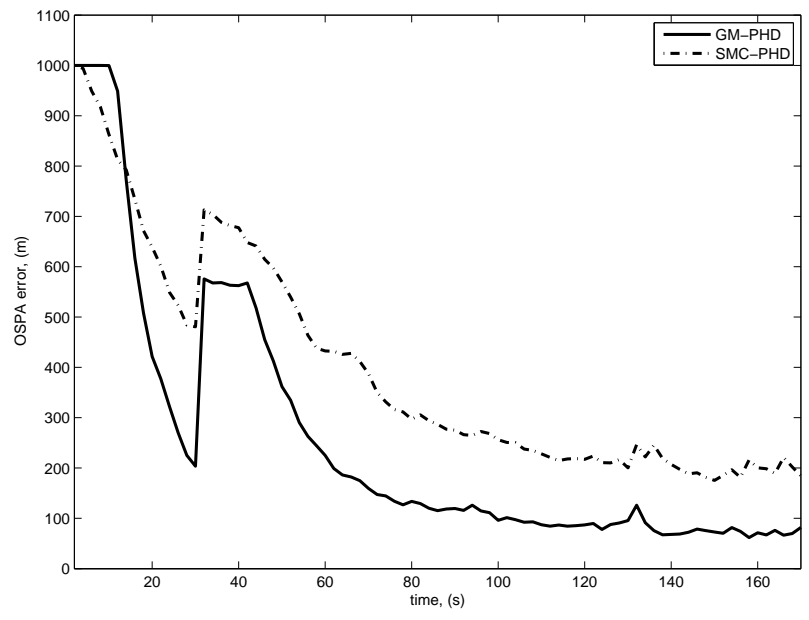

Figure 4: Average OSPA error over time. GM-PHD (solid line), SMC-PHD (dash-dot line).

truth state set $Y=\left\{\mathbf{y}_{1}, \ldots \mathbf{y}_{m}\right\}$ for $0<n \leq m$ is;

$$
d_{p, c}^{O S P A}(X, Y)=\left(\min _{\pi} \frac{1}{m} \sum_{i=1}^{n} d_{c}\left(\mathbf{x}_{i}, \mathbf{y}_{\pi i}\right)^{p}+\frac{c^{p}}{m} \cdot(m-n)\right)^{1 / p}
$$

where $c>0$ is the cut-off parameter, $d(\mathbf{x}, \mathbf{y})=\|\mathbf{x}-\mathbf{y}\|$ is the distance between single-target state vectors $\mathbf{x}$ and $\mathbf{y}, p \geq 1$ is a unitless real number, $d_{c}(\mathbf{x}, \mathbf{y})=\min \{c, d(\mathbf{x}, \mathbf{y})\}$ is the cut-off metric associated with $d(\mathbf{x}, \mathbf{y})$. The summation is taken over all permutations $\pi$ on $1, \ldots, m$. If $n=0$ and $n \leq m$ then $d_{p, c}^{O S P A}(\varnothing, Y)=c$. If $n>m$ then $d_{p, c}^{O S P A}(X, Y)=d_{p, c}^{O S P A}(Y, X)$ and also $d_{p, c}^{O S P A}(\varnothing, \varnothing)=0$. In this work, we use parameters $c=1000 \mathrm{~m}$ and $p=1$ so that OSPA distance represents the sum of the localization error and the cardinality error. The cut-off parameter $c$ determines the relative weighting of the penalties assigned to localization and cardinality errors.

Single run of each filter is shown in Figs.2(a),2(b). Moreover, performance results of the GM-PHD and SMC-PHD filters, in terms of OSPA error, are presented in Fig. 4. OSPA error is averaged over 1000 Monte Carlo runs. As can be seen from the results, both filters successfully detect and track two targets. However, almost for all time instances, the GM-PHD filter gives better results than the SMC-PHD filter. Initially it takes sometime for both filters to detect the presence of a target. Therefore, the OSPA error is dominated by cardinality error, $c$, as expected. During the birth of the second target, at time $t=30 \mathrm{~s}$, and during the death of the first target, at time $t=130$ s, again OSPA error is dominated by the cardinality error. For the given configurations, averaged computational time of a single run (i.e. a $T_{\text {sim }}$ long scenario) for the GM-PHD and SMC-PHD filter are $C T_{G M}=0.32 \mathrm{~s}$ and $C T_{S M C}=5.86 \mathrm{~s}$, respectively on a regular desktop. As expected, computational time required for the GM-PHD is much less than what SMCPHD requires. Also note that, this time gap will substantially increase for higher number of targets [37]. Using more particles improves the performance of the SMC-PHD filter up to a certain accuracy. However, computation time will increase. In Table 1, we provide some results showing the relation between number of particles, computational time for a single run and averaged OSPA error at a certain time.

Table 1: OSPA error and computational times (CT) for a single run for different number of particle numbers

\begin{tabular}{ccc}
\hline \hline \# of particles & $C T$ & OSPA(at $k . \Delta=100 \mathrm{~s})$ \\
\hline 4000 & 1.4 & 444 \\
8000 & 3.23 & 333 \\
12000 & 4.67 & 285 \\
16000 & 5.86 & 256 \\
20000 & 7.45 & 205
\end{tabular}

It is important to note once again that Doppler-only tracking is a challenging problem due following points; First of all, target state remains unobservable before collecting at least three Doppler measurements from sensors with different locations, because Doppler shift information alone is not informative enough. Therefore, since newborn target intensity covers whole surveillance area it takes some time to detect targets by both filters. Secondly, since Doppler shift measurements are typically accurate and initially target state is unobservable, initial measurement updates are weighted significantly. Meaning that sample impoverishment becomes a serious problem for SMC implementation and should be handled. There exist techniques in the literature proposed for this problem [27], [26]. However, careful parameter fine tuning is necessary and it is not always straightforward. On the other hand, in GM implementation no such problem exist and implementation is easier. Lastly, handling these two problems get worse if the prior newborn intensity covers whole surveillance volume, which is the case in this work [20], [19], [21], [22].

To sum up, for the considered application, we found the GMPHD filter is more intuitive, easy to implement, effective and much more computationally efficient than the SMC-PHD filter. EKF based adaptation of the GM-PHD filter makes it possible to apply scenarios where targets have non-linear complex motion and measurement models [16], [33], [34], [35], [36], [37]. Number of Gaussian components required for GM-PHD filter is much less than the number of particles used in SMC-PHD filter. Moreover, as the number of targets increases, more particles are needed for SMC-PHD filter to provide acceptable accuracy. A similar comparison work can also be found in [37].

\section{Acoustic Field Trials}

In this section, we present the performance results of the GM-PHD and SMC-PHD filters in tracking multiple ground targets using acoustic reflections. For that purpose, in the following subsections we provide some important points regarding the experimental setup and acoustic measurements [16].

\subsection{The Experimental Setup}

An acoustic experiment is conducted to obtain Doppler data for numerical evaluation. A Dodge van passes a loudspeaker 


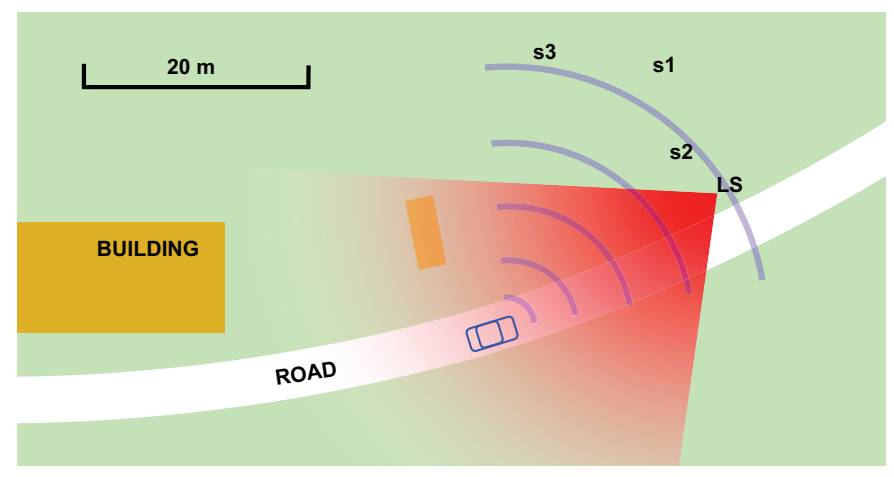

Figure 5: Experimental setup. A directive loudspeaker emits a $10 \mathrm{kHz}$ tone that is reflected by vehicles passing on the road. Red indicates the main sound field direction. The reflected sound, here illustrated by blue arcs, is in turn picked up by 3 microphones, $s 1, \mathrm{~s} 2$, and $\mathrm{s} 3$. When the vehicle is moving, the reflected sound includes Doppler components that are filtered and used in the tracking.

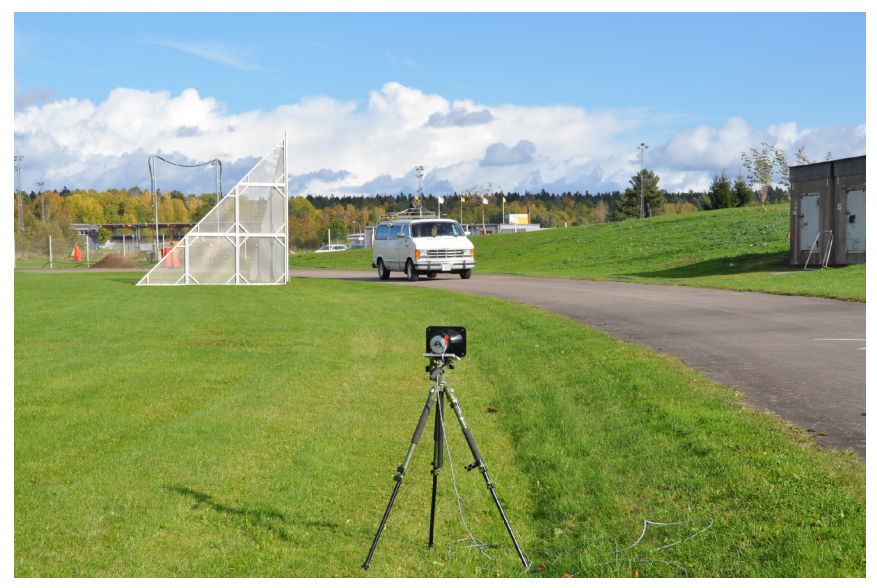

Figure 6: Dodge van approaching the loudspeaker. (The photograph was taken when the loudspeaker was directed opposite to what is showed in Fig.5.)

and microphone rig at velocities in the range $20-40 \mathrm{~km} / \mathrm{h}$, see Fig.6. Three high-end microphones are deployed on tripods $1.60 \mathrm{~m}$ above ground and with $10-16 \mathrm{~m}$ spacing, see Fig.5. The LS, an Eighteen Sound XD125 high frequency driver, with a horizontal beam width of somewhat less than $90^{\circ}$, gives a 10 $\mathrm{kHz}$ sinusoid at $129 \mathrm{~dB}$ SPL (that is, dB SPL, logarithmic sound pressure level $1 \mathrm{~m}$ in front of the speaker and with respect to the mean square of $20 \mu \mathrm{Pa}$.) The target is acoustically passive, in other words it is only the reflected sound from the LS that is detected and used in the tracking.

As a localization and tracking method, the described Doppler-by-sound-reflection has perhaps limited applications due to the high loudspeaker sound pressure in fact needed. Certainly, the experiment could have been designed in other ways, but recall that the motivation for the tracking technique in focus is that it in the future will be used with (narrow-band) radio transmitters of opportunity, and that we have used the acoustics as a first step to prove the tracking concept.

\subsection{Acoustic Measurements and Models}

At the trial, the acoustic background noise level is rather high due to nearby construction work and traffic; around 60-70 dB.

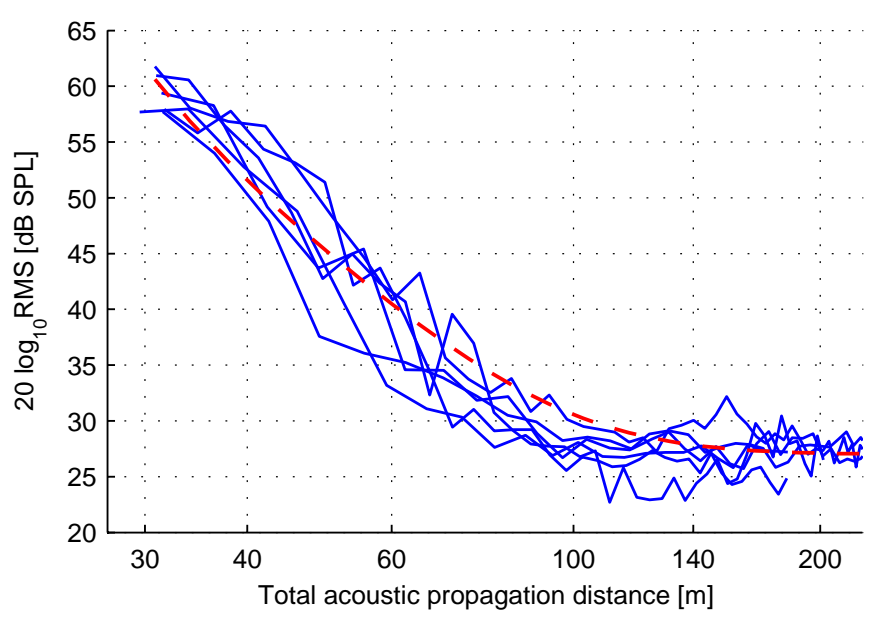

Figure 7: Acoustic SPL decay with total propagation distance, both in logarithmic scale. Aimed for ease of interpretation, the total distance adds up the two partial distances LS/target and target/microphone. The solid curves (blue) are acoustic (narrow band) power as observed at $s_{3}$ for six target passages. The dashed curve (red) corresponds to the theoretical decay (53), assuming a constant reflection loss of $-20 \mathrm{~dB}$ and an atmospheric attenuation of $-0.2 \mathrm{~dB} / \mathrm{m}$.

The Doppler detection is however restricted to the frequencies just above and below the $10 \mathrm{kHz}$ tone, frequency regions we define as $10 \mathrm{k} \pm[100800] \mathrm{Hz}$. The noise contributions in these frequency regions sum up to around $10 \mathrm{~dB}$. When the LS is switched on, the noise level rises to $27 \mathrm{~dB}$ (with no target present), so $17 \mathrm{~dB}$ noise happens to be self-induced and attributable to non-linear paths and driver imperfections (speculatively).

A rudimentary and macroscopic model for the received signal strength at microphone $s_{i}$ would be

$$
E_{k, i}=E_{0}+G_{k}+G_{r}+G_{k, i}+G_{a} \quad[\mathrm{~dB}]
$$

where $E_{0}$ is the emitted power defined $1 \mathrm{~m}$ in front of the source LS, $G_{k}$ the spheric (free field) loss between LS and target $k, G_{k, i}$ likewise for target $k$ and microphone $i, G_{r}$ the constant target reflection loss, and $G_{a}$ the atmospheric attenuation;

$$
\begin{aligned}
G_{k} & =-20 \log _{10} d_{k}^{t}, \\
G_{k, i} & =-20 \log _{10} d_{k, i}, \\
G_{a} & =-\xi\left(d_{k}^{t}+d_{k, i}\right),
\end{aligned}
$$

for the atmospheric parameter $\xi$. $\xi$ depends on factors like the air humidity and temperature but also on acoustic frequency. At $10 \mathrm{kHz}, \xi$ typically varies between 0.1 and $0.3 \mathrm{~dB} / \mathrm{m}$. As before, $d_{k}^{t}$ and $d_{k, i}$ are the LS/target/microphone distances. The term $G_{k}$ here accounts for the power loss as the sound reflects in various surfaces on the target. These reflection surfaces are not trivial to identify, and thus it is difficult to assign a prior value to $G_{k}$. Modeling the reflection from a vehicle in motion as a constant attenuation in this way is admittedly a grand simplification, but quantitatively it may provide us with the right order of magnitude. In Fig.7, the loss model (53) is validated on 6 passages with the van. It is concluded that the real path is at least as destructive as the model predicts. To fit the model to data, we have identified the reflection loss as $G_{r}=-20 \mathrm{~dB}$. 


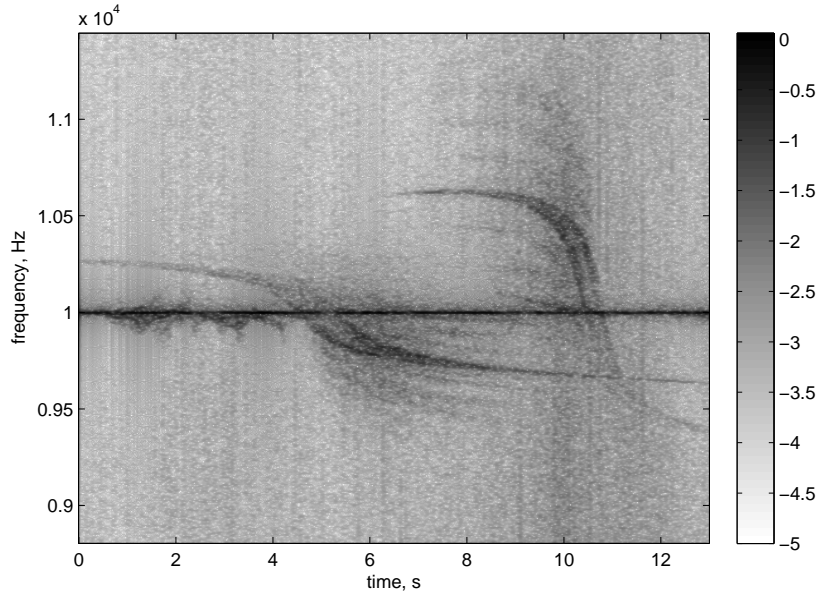

Figure 8: Time-frequency diagram of the microphone-1 output signal obtained by STFT. Carrier frequency, $f_{c}=10 \mathrm{kHz}$.

In summary, the signal-to-noise ratio (SNR) is, in our experimental setup, fairly well described by

$$
\mathrm{SNR}_{i, k}=E_{k, i}-E_{n}
$$

with the background noise $E_{n}=27 \mathrm{~dB}, \quad E_{0}=129 \mathrm{~dB}$, $\xi=0.2 \mathrm{~dB} / \mathrm{m}$, and $G_{r}=-20 \mathrm{~dB}$. Since already $129 \mathrm{~dB}$ source power is impractically loud in most situations, it is noted that $2 \times 50 \mathrm{~m}$ total distance would be close to the range limit for acoustic Doppler radar, at least in the case with a narrow-band $10 \mathrm{kHz}$ source. Over $2 \times 50 \mathrm{~m}$ the received signal strength essentially falls below the noise floor and the Doppler frequency tracking becomes unreliable.

Short-time Fourier transform (STFT) is used to get the timefrequency diagram of microphone outputs and detection is performed in the time-frequency domain. STFT of the first microphone is seen in Fig.8. Peaks of the STFT that exceed a threshold level are collected as measurements. However, different kinds of instantaneous frequency estimation techniques can also be adapted to have better detection results [41], [42], [43]. Doppler-shifts corresponding to two vehicles around the carrier frequency can be distinguished in the time-frequency domain. Depending on the look direction of the LS and field-ofview (FOV) of the microphones, SNR of the reflections vary in time. Moreover, strong harmonics appear around the fundamental frequencies, which have negative effect on tracking performance.

\subsection{The PHD Filtering Results}

In this subsection, we present performance results of both the GM-PHD and SMC-PHD filters in tracking 2 vehicles using noisy Doppler shift measurements of separately distributed 3 microphones. Total duration of the experiment is $13 \mathrm{~s}$. Vehicle1 starts its motion at time $k=0 \mathrm{~s}$, position $[78,72.8] \mathrm{m}$ and dies at time $k=13 \mathrm{~s}$, position $[9.76,43.9] \mathrm{m}$. Vehicle-2 is born at time $k=7.5 \mathrm{~s}$, position $[17.2,46.7] \mathrm{m}$ and dies at time $k=13 \mathrm{~s}$, position $[76.5,73.3] \mathrm{m}$. True vehicle trajectories are plotted in

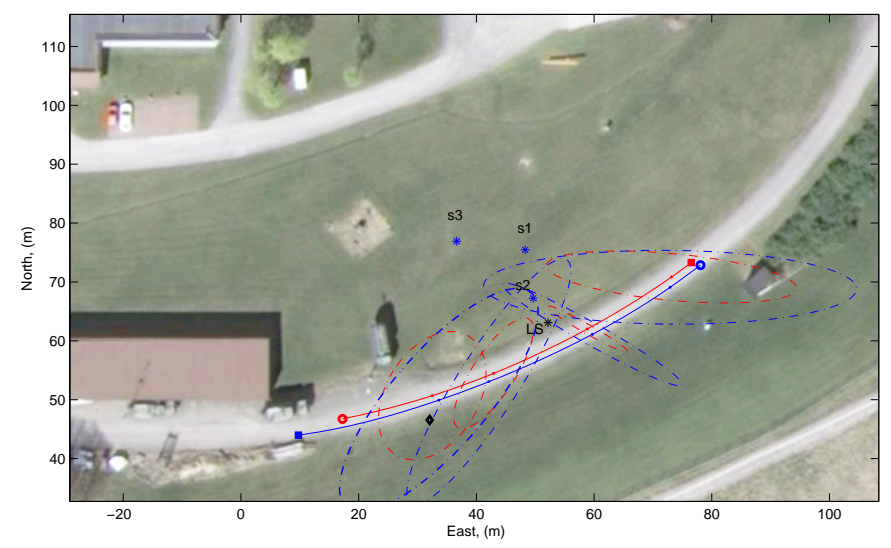

Figure 9: Map of the experiment area. Blue stars $(*)$ are for microphones $(\mathrm{s} 1, \mathrm{~s} 2, \mathrm{~s} 3)$, black star $(*)$ is for the LS and black $\diamond$ is the look direction of the LS. $\circ$ : positions at which vehicles are born and $\square$ : positions at which vehicles die. Blue and red lines are the vehicle- 1 and the vehicle- 2 trajectories, respectively. Blue and red dash-dot ellipsoids are the localization accuracies with a $90 \%$ confidence interval corresponding to vehicle-1 and the vehicle-2, respectively, at some position points represented with dots.

Fig.9. On the figure, blue and red lines denote the vehicle trajectories of the vehicle- 1 and vehicle-2, respectively. The symbol 'circle' represents locations at which vehicles are born and the symbol 'square' represents locations at which vehicles die. Blue stars are for microphone positions and black star is for LS location. The black symbol 'diamond' is the look direction of the LS. Static localization accuracies, which is the inverse of the Fisher information matrix, corresponding to each target at certain points, calculated by $\left(\left(\sum_{i=1}^{3} \frac{\mathbf{H}_{i}^{T} \mathbf{H}_{i}}{\sigma_{\varepsilon}^{2}}\right)^{-1}\right)$, are also plotted on the same figure [44].

Some common parameters used by the GM-PHD and SMCPHD filters are: $\Delta=85 \mathrm{~ms}, \sigma_{v}=4 \mathrm{~m} / \mathrm{s}^{2}, p_{D}=0.79, p_{s}=0.98$ and $\sigma_{\varepsilon}=5 \mathrm{~Hz}$. The clutter RFS, $K_{k}$, follows a uniform Poisson model over the surveillance region $[-800,800] \mathrm{Hz}$, with an average number of clutter returns per unit region, $\gamma_{c}=$ $1 \times 10^{-3} \mathrm{~Hz}^{-1}$. In a similar way as described in Section 6 and considering the FOV of the microphones, relative position of the road segment and the two-way acoustic path attenuation, newborn target intensity $b(\mathbf{x} \mid z)$ is determined. In other words, road segment is covered with several Gaussian distributions and formed GM is used as $b(\mathbf{x} \mid z)$. Maximum number of Gaussian components for GM-PHD is set to $J_{\max }=70$. Number of particles per persistent target is $M_{p}=8000$ and for each measurement $M_{b}=8000$ particles are generated by drawing from $b(\mathbf{x} \mid z)$.

Tracking results of both filters are presented in Figure 10. At some time instants, short discontinuities occur in the tracks. This is due to the harmonics of the fundamental acoustic reflection and having less number of measurements around carrier frequency. Note that, during the transient regions where the sign of the Doppler shifts change, vehicles behave like extended targets and produce more than one measurement. This effect should be taken care of for better accuracy. It is seen from the figures that both filters detects and tracks position and velocity of the vehicles from only Doppler shift measurements. 

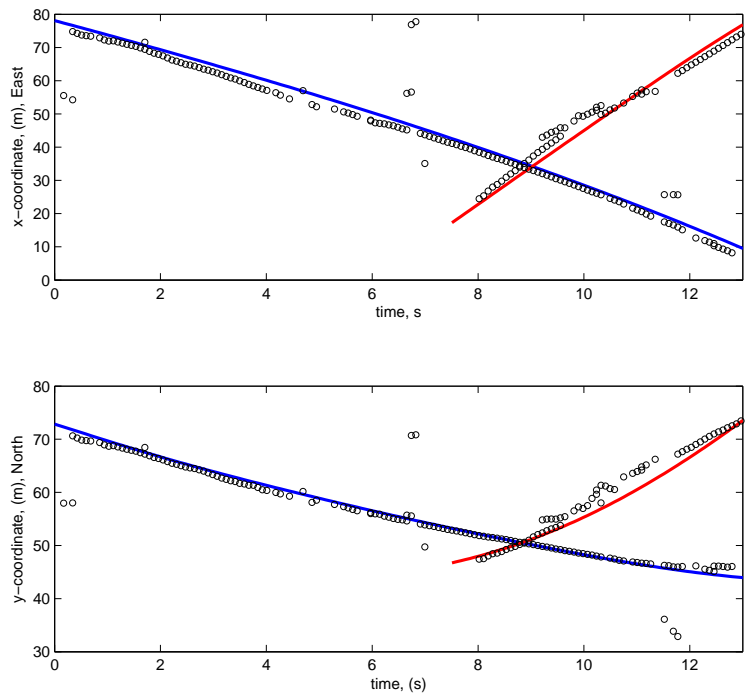

(a) Position estimates of the GM-PHD filter

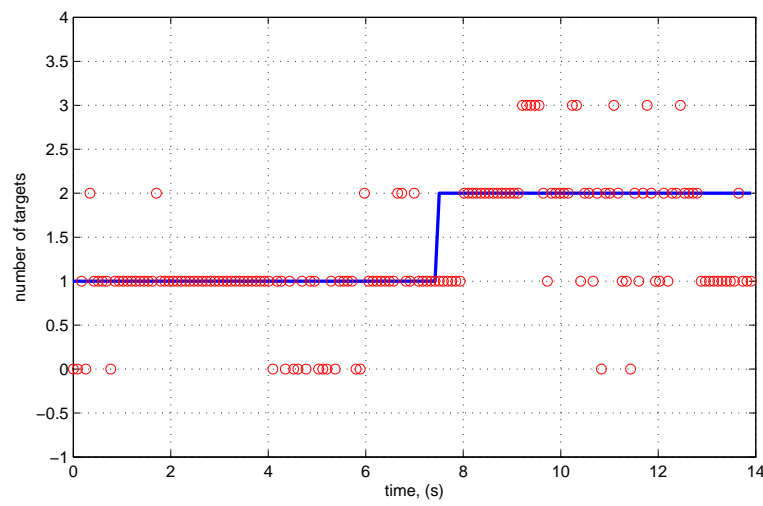

(c) Number of vehicle estimates of the GM-PHD filter.
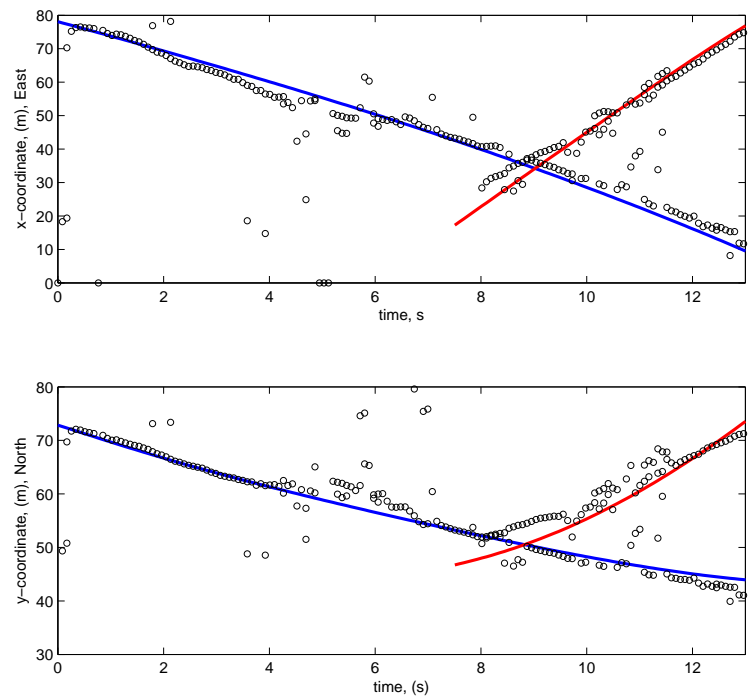

(b) Position estimates of the SMC-PHD filter.

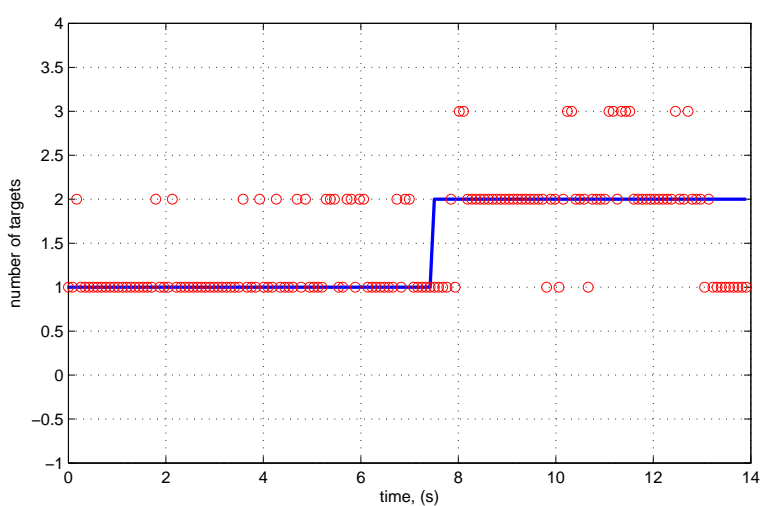

(d) Number of vehicle estimates of the SMC-PHD filter.

Figure 10: Position and number of vehicle estimates of the GM-PHD and SMC-PHD filters using acoustic Doppler measurements. Black and red circles represent position and number of vehicles estimates, respectively. Blue and red solid lines denote true values.

However, GM-PHD filter provides better tracking results both in location and cardinality estimates.

As a last word, observability of targets is a problem in target tracking when only Doppler measurements are used. However, this problem can be alleviated by systematically placing sensors with respect to the transmitter. Therefore, we believe that, with a better microphone placement around road, tracking results will significantly improve. Moreover, it could be safely said that the off-road tracking of multiple vehicles is possible using a carefully located microphone sensor network covering the area in consideration.

\section{Conclusion}

In this paper, we studied the problem of multiple target tracking over a network of separately located Doppler-shift measuring sensors. For this challenging problem, we used both
SMC-PHD and GM-PHD filters. Simulation results show that both PHD filter implementations successfully tracks multiple targets using only Doppler shift measurements. Moreover, performance of the GM-PHD and SMC-PHD filters are also tested on real acoustic measurements. Experimental study results reveal that it is possible to track multiple ground targets using acoustic Doppler shift measurements in a passive multi-static scenario. We observed that the GM implementation of the PHD filter is both more effective and easier to implement than the SMC-PHD. A future direction would be to increase the coverage of the passive system by adding more microphones systematically to be able to cover a longer segment of the road and get more accurate and robust results. Moreover, it is also of interest to us to define other acoustic waveforms that do are more pleasant to hear and to use wider bands that decrease the sound level requirements. 


\section{Acknowledgment}

The authors gratefully acknowledge fundings from the ELLIIT joint research program, Swedish Foundation for Strategic Research (SSF) in the center MOVIII and the Swedish Defence Research Agency (FOI) Center for Advanced Sensors, Multisensors and Sensor Networks (FOCUS) funded by the Swedish Governmental Agency for Innovation Systems (VINNOVA).

\section{References}

[1] M. C. (Editor), Bistatic Radars: Emerging Technology, John Wiley, 2008.

[2] H. D. Griffiths, C. J. Baker, Passive coherent location radar systems. part 1: Performance prediction, IET Radar Sonar Navigation 3 (152) (2005) 153-159.

[3] K. Chetty, K. Woodbridge, H. Guo, G. Smith, Passive bistatic WiMAX radar for marine surveillance, in: IEEE Radar Conference, 2010.

[4] M. Daun, U. Nickel, Tracking in multistatic passive radar systems using DAB/DVB-T illumination, Signal Process. 92 (6) (2012) 1365-1386.

[5] D. K. P. Tan, H. Sun, Y. Lu, M. Lesturgie, H. L. Chan, Passive radar using global system for mobile communication signal: theory, implementation and measurements, IET Radar Sonar Navigation 3 (152) (2005) 116-123.

[6] P. Krysik, K. Kulpa, P. Samczynski, K. Szumski, J. Misiurewicz, Moving target detection and imaging using GSM-based passive radar, in: IET Int. Conference on Radar Systems, 2012.

[7] R. Zemmari, M. Daun, M. Feldmann, U. Nickel, Maritime surveillance with GSM passive radar: Detection and tracking of small agile targets, in: IRS Int. Radar Symposium, 2013.

[8] L. R. Malling, Radio Doppler effect for aircraft speed measurements, IRE Proc. IRE 35 (11) (1947) 1357-1360.

[9] W. R. Fried, Principles and performance analysis of Doppler navigation systems, IRE Trans. on Aeronautical and Navigational Electronics 4 (4) (1957) 176-196.

[10] S. N. Salinger, J. J. Brandstatter, Application of recursive estimation and Kalman filtering to Doppler tracking, IEEE Trans. Aerosp. Electron. Syst. 4 (4) (1970) 585-592.

[11] D. C. Torney, Localization and observability of aircraft via Doppler shifts, IEEE Trans. Aerosp. Electron. Syst. 43 (3) (2007) 1163-1168.

[12] Y. C. Xiao, P. Wei, T. Yuan, Observability and performance analysis of bi/multi-static Doppler-only radar, IEEE Trans. Aerosp. Electron. Syst. 46 (4) (2010) 1654-1667.

[13] A. N. Bishop, M. Smith, Remarks on the Cramer-Rao inequality for Doppler-based target parameter estimation, in: ISSNIP Int. Conf. Intelligent Sensors, Sensor Networks and Inf. Process. (ISSNIP), 2010.

[14] I. Shames, A. N. Bishop, M. Smith, B. D. O. Anderson, Doppler-shift target localization, IEEE Trans. Aerosp. Electron. Syst. 49 (1) (2013) 266276.

[15] A. Amar, A. J. Weiss, Localization of narrowband radio emitters based on Doppler frequency shifts, IEEE Trans. Signal Process. 56 (11) (2008) 5500-5508.

[16] M. B. Guldogan, D. Lindgren, F. Gustafsson, H. Habberstad, U. Orguner, Multiple target tracking with Gaussian mixture PHD filter using passive acoustic Doppler-only measurements, in: IEEE Int. Conf. Information Fusion (FUSION), 2012.

[17] D. Lindgren, M. B. Guldogan, F. Gustafsson, H. Habberstad, G. Hendeby, Acoustic source localization in a network of Doppler shift sensors, in: IEEE Int. Conf. Information Fusion (FUSION), 2013.

[18] M. B. Guldogan, U. Orguner, F. Gustafsson, Gaussian mixture PHD filter for multi-target tracking using passive Doppler-only measurements, in: IET Data Fusion \& Tracking Conf., 2012.

[19] B. Ristic, A. Farina, Target tracking via multi-static Doppler shifts, IET Radar Sonar Navigation 7 (5) (2013) 508-516.

[20] B. Ristic, A. Farina, Recursive bayesian state estimation from Dopplershift measurements, in: ISSNIP Int. Conf. Intelligent Sensors, Sensor Networks and Inf. Process. (ISSNIP), 2011.

[21] C. Fantacci, G. Battistelli, L. Chisci, A. Farina, A. Graziano, Multiplemodel algorithms for distributed tracking of a maneuvering target, in: IEEE Int. Conf. Information Fusion (FUSION), 2012.
[22] R. J. Webster, An exact trajectory solution from Doppler shift measurements, IEEE Trans. Aerosp. Electron. Syst. 18 (2) (1982) 249-252.

[23] R. P. S. Mahler, Statistical multisource-multitarget information fusion, Artech House, 2007.

[24] R. Mahler, Multitarget Bayes filtering via first-order multitarget moments, IEEE Trans. Aerosp. Electron. Syst. 39 (4) (2003) 1152-1178.

[25] B. N. Vo, W. K. Ma, The Gaussian mixture probability hypothesis density filter, IEEE Trans. Signal Process. 54 (11) (2006) 4091-4104.

[26] M. S. Arulampalam, S. Maskell, N. Gordon, T. Clapp, A tutorial on particle filters for on-line nonlinear/non-Gaussian Bayesian tracking, IEEE Trans. Signal Process. 50 (2) (2002) 174-188.

[27] B. Ristic, S. Arulampalam, N. Gordon, Beyond the Kalman filter: Particle filters for tracking applications, Artech House, 2007.

[28] F. Gustafsson, Particle filter theory and practice with positioning applications, IEEE Aerosp. Electron. Syst. Mag. 25 (7) (2010) 53-82.

[29] Y. Bar-Shalom, X. R. Li, T. Kirubarajan, Estimation with Applications to Tracking and Navigation, John Wiley, 2001.

[30] B. Ristic, D. Clark, B. Vo, Improved SMC implementation of the PHD filter, in: IEEE Int. Conf. Information Fusion (FUSION), 2010.

[31] B. N. Vo, S. Singh, A. Doucet, Sequential Monte Carlo methods for multitarget filtering with random finite sets, IEEE Trans. Aerosp. Electron. Syst. 41 (4) (2005) 1224-1245.

[32] B. Ristic, D. Clark, B. Vo, B. Vo, Adaptive target birth intensity for PHD and CPHD filters, IEEE Trans. Aerosp. Electron. Syst. 48 (2) (2012) 1656-1668

[33] D. Clark, B. N. Vo, J. Bell, GM-PHD filter multi-target tracking in sonar images, in: SPIE Proc. SPIE Defense and Security Symposium, 2006.

[34] M. Ulmke, O. Erdinc, P. Willett, GMTI tracking via Gaussian mixture cardinalized probability hypothesis density filter, IEEE Trans. Aerosp. Electron. Syst. 46 (4) (2010) 1821-1833.

[35] D. Laneuville, J. Houssineau, Passive multi target tracking with GM-PHD filter, in: IEEE Int. Conf. Information Fusion (FUSION), 2010.

[36] R. Georgescu, P. Willett, The GM-CPHD tracker applied to real and realistic multistatic sonar data sets, IEEE J. Ocean. Eng. 37 (2) (2012) 220235.

[37] M. Pace, Comparison of PHD based filters for the tracking of 3D aerial and naval scenarios, in: IEEE Radar Conference, 2010.

[38] P. E. Howland, Target tracking using television-based bistatic radar, IET Radar Sonar Navigation 146 (3) (1999) 166-174.

[39] P. E. Howland, D. Maksimiuk, G. Reitsma, FM radio based bistatic radar, IET Radar Sonar Navigation 152 (3) (2005) 107-115.

[40] D. Schuhmacher, B. T. Vo, B. N. Vo, A consistent metric for performance evaluation of multi-object filters, IEEE Trans. Signal Process. 56 (8) (2008) 3447-3457.

[41] B. Boashash, Estimating and interpreting the instantaneous frequency of a signal. II. algorithms and applications, Proc. IEEE 80 (4) (1992) 540-568.

[42] I. Djurovic, L. Stankovic, An algorithm for the Wigner distribution based instantaneous frequency estimation in a high noise environment, Signal Process. 84 (3) (2004) 631-643.

[43] E. Sejdic, I. Djurovic, J. Jianga, Timefrequency feature representation using energy concentration: An overview of recent advances, Digital Signal Process. 19 (1) (2009) 153-183.

[44] S. M. Kay, Fundamentals of Statistical Signal Processing, Volume I: Estimation Theory, Prentice Hall, 1993. 